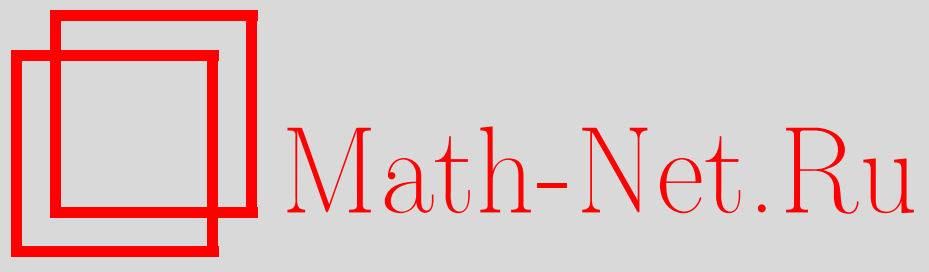

В. В. Мещеряков, Универсальные операторы Данкла, УМH, 2009, том 64, выпуск 1, 155-156

DOI: https://doi.org/10.4213/rm9248

Использование Общероссийского математического портала Math-Net.Ru подразумевает, что вы прочитали и согласны с пользовательским соглашением http: //www . mathnet.ru/rus/agreement

Параметры загрузки:

IP: 54.205 .225 .156

26 апреля 2023 г., 13:29:18

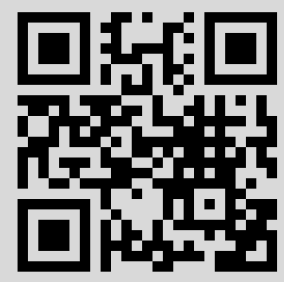




\section{Универсальные операторы Данкла}

\section{В. В. Мещеряков}

1. Введение. Классические операторы Данкла рационального типа, ассоциированные с системой корней $R$, определяются следующей формулой [1]: $T_{\xi}=\partial_{\xi}-$ $\sum_{\alpha \in R_{+}} k_{\alpha}(\alpha \mid \xi) \frac{s_{\alpha}}{(x \mid \alpha)}$, где $\xi$ - вектор пространства $V$, которое порождается системой корней $R ; \partial_{\xi}$ - дифференцирование в направлении $\xi ; R_{+}-$подсистема положительных корней; $k_{\alpha}$ - целые числа, зависящие от длины вектора $\alpha ;(\cdot \mid \cdot)$ - скалярное произведение в $V ; s_{\alpha}$ - отражение в $V$ относительно гиперплоскости, ортогональной к $\alpha$. На функциях $f$ отражение $s_{\alpha}$ действует по формуле $\left(s_{\alpha} f\right)(x)=f\left(s_{\alpha} x\right)$.

В работе [2] приводятся обобщения операторов Данкла. Там же установлены свойства, которые являются полными аналогами классических на так называемых многообразиях Бете и Данкла (подробнее см. п. 2).

Целью статьи является описание многообразий Бете и Данкла.

2. Многообразия Бете и Данкла. Для каждой приведенной неприводимой системы корней существует единственная инвариантная невырожденная симметрическая билинейная форма $F_{R}(x, y)$ на $V$ [3], удовлетворяющая условию $F_{R}(x, y)=$ $\sum_{\alpha \in R_{+}} F_{R}(x, \alpha) F_{R}(\alpha, y)$

Рассмотрим комплексное пространство $\mathbb{C}^{N}, N=\# R_{+}$. В $\mathbb{C}^{N}$ можно ввести координаты $u=\left\{u_{s_{\alpha}}, \alpha \in R_{+}\right\}$.

Действие группы Кокстера $W(R)$ на пространстве комплекснозначных функций на $\mathbb{C}^{N}$ определим формулой $w f\left(u_{s_{\alpha}}\right)=f\left(\varepsilon_{w}(\alpha) u_{w s_{\alpha} w^{-1}}\right)$, где $\varepsilon_{w}(\alpha)=1$, если $w \alpha \in R_{+}$, и $\varepsilon_{w}(\alpha)=-1$ в противном случае.

Рассмотрим оператор $D_{\gamma}=\sum_{\alpha \in R_{+}} F_{R}(\gamma, \alpha) \frac{\partial}{\partial u_{s_{\alpha}}}$ и для всех $\gamma \in R_{+}$определим "универсальные" операторы Данкла $\nabla_{\gamma}=-D_{\gamma}+\sum_{\alpha \in R_{+}} \frac{F_{R}(\gamma, \alpha) k_{\alpha}}{u_{s_{\alpha}}} s_{\alpha}$ и "универсальный" гамильтониан типа Калоджеро $H_{C}=-\widehat{\Delta}+\sum_{\alpha \in R_{+}} \frac{F_{R}(\alpha, \alpha)\left(k_{\alpha}^{2}-k_{\alpha} s_{\alpha}\right)}{u_{s_{\alpha}}^{2}}, \widehat{\Delta}=\sum_{\gamma \in R_{+}} D_{\gamma}^{2}$.

Подмножество в $\mathbb{C}^{N}$, на котором операторы $\nabla_{\gamma}$ коммутируют, называется многообразием Данкла. В [2] показано, что многообразие Данкла определяется системой

$$
\sum_{\alpha, \beta \in R_{+}: s_{\alpha} s_{\beta}=w \neq 1} k_{\alpha} k_{\beta} \frac{F_{R}(\gamma, \alpha) F_{R}(\delta, \beta)-F_{R}(\gamma, \beta) F_{R}(\delta, \alpha)}{u_{s_{\alpha}} u_{s_{\beta}}}=0, \quad w \in W(R) .
$$

Подмножество в $\mathbb{C}^{N}$, где сумма квадратов операторов $\nabla_{\gamma}$ совпадает с $H_{C}$, называется многообразием Бете. Оно определяется системой

$$
\sum_{\alpha, \beta \in R_{+}: s_{\alpha} s_{\beta}=w \neq 1} k_{\alpha} k_{\beta} \frac{F_{R}(\alpha, \beta)}{u_{s_{\alpha}} u_{s_{\beta}}}=0, \quad w \in W(R) .
$$

3. Основные результаты. Далее будут использоваться обозначения работы [4].

Теорема 1. Многообразия Бете и Данкла совпадают.

Поэтому многообразия Бете и Данкла назовем многообразием Бете-Данкла.

Из результатов в [4] и теоремы 1 вытекают следующие утверждения.

Работа выполнена при поддержке РФФИ (грант № 07-01-00085). 
Теорема 2. Многообразие Бете-Данкла, ассоииированное с системой корней типа $A_{n-1}$, есть плоскость $u_{1 j}-u_{1 k}+u_{j k}=0,1<j<k \leqslant n$, размерности $n-1$ в пространстве $\mathbb{C}^{n(n-1) / 2}$ с исключенными координатными гиперплоскостями $u_{i j}=0$, где $1 \leqslant i<j \leqslant n$.

Теорема 3. Многообразие Бете-Данкла, ассоциированное с системой корней типа $D_{n}$, есть п-мерная плоскость, определяемая системой уравнений

$$
\begin{cases}u_{i j}-v_{i, j+1}+v_{j, j+1}=0, & 1 \leqslant i<j<n, \\ u_{i n}-v_{i, n-1}+v_{n-1, n}=0, & 1 \leqslant i<n-1, \\ u_{n-1, n}-v_{n-2, n-1}+v_{n-2, n}=0, & \\ v_{i, i+1}-v_{i, i+2}-v_{i+1, i+3}+v_{i+2, i+3}=0, & 1 \leqslant i<n-2, \\ v_{i j}-v_{i, j+1}-v_{i+1, j}+v_{i+1, j+1}=0, & 1 \leqslant i<j-1, \quad j<n,\end{cases}
$$

на множестве $\tilde{X}_{D}=\mathbb{C}^{n(n-1)} \backslash \bigcup_{1 \leqslant i<j \leqslant n}\left\{u_{i j}=0, v_{i j}=0\right\}$.

Теорема 4. Многообразие Бете-Данкла, ассоииированное с системой корней типа $B_{n}$, является поверхностъю размерности $n+1$ во множестве $\widetilde{X}_{B_{n}}=\mathbb{C}^{n^{2}}$ । $\bigcup_{1 \leqslant i<j \leqslant n}\left\{u_{i j}=0, v_{i j}=0, w_{i}=0\right\}$ и определяется системой, являющейся обвединением системы (1) и уравнений $u_{i j}\left(w_{i}+w_{j}\right)=v_{i j}\left(w_{i}-w_{j}\right), 1 \leqslant i<j \leqslant n$.

При $n=2$ система состоит из одного уравнения $u_{12}\left(w_{1}+w_{2}\right)=v_{12}\left(w_{1}-w_{2}\right)$, которое задает многообразие Сегре [5].

УтвеРждениЕ 1. Многообразие Бете-Данкла, ассоциированное с системой корней типа $B_{2}$, является многообразием Сегре.

\section{4. Примеры “универсальных" операторов Данкла.}

СлучАй А. На многообразии Бете-Данкла, ассоциированном с системой корней типа $A_{n-1}$, положим $u_{i j}=x_{i}-x_{j}, 1 \leqslant i \leqslant j \leqslant n$. В новых координатах операторы $\nabla_{\gamma}$ будут иметь вид $\nabla_{\gamma}=-\partial_{\gamma}+\sum_{i<j} \frac{k_{\alpha_{i j}}}{n}\left(\gamma \mid \alpha_{i j}\right) \frac{s_{\alpha_{i j}}}{x_{i}-x_{j}}$.

СлучАй D. Для системы корней типа $D_{n}$ на многообразии Бете-Данкла можно ввести координаты $u_{i j}=x_{i}-x_{j}, v_{i j}=x_{i}+x_{j}, 1 \leqslant i<j \leqslant n$, в которых

$$
\nabla_{\gamma}=-\partial_{\gamma}+\sum_{i<j} \frac{k_{\alpha_{i j}}}{2(n-1)}\left(\gamma \mid \alpha_{i j}\right) \frac{s_{\alpha_{i j}}}{x_{i}-x_{j}}+\sum_{i<j} \frac{k_{\beta_{i j}}}{2(n-1)}\left(\gamma \mid \beta_{i j}\right) \frac{s_{\beta_{i j}}}{x_{i}+x_{j}} .
$$

УтвеРЖДЕниЕ 2. Ограничения "универсальных" операторов Данкла, ассоциированных с системой корней типа $A_{n-1}$ или $D_{n}$, на многообразие Бете-Данкла совпадают с рациональными операторами Данкла.

СлучАй В. Для системы корней типа $B_{n}$ классические операторы получаются при ограничении на некоторое линейное подмножество.

УтвеРЖДЕнИЕ 3. Ограничения операторов Данкла $\nabla_{\gamma}$, ассоциированных с системой корней типа $B_{n}$ на плоскость $u_{i j}=w_{i}-w_{j}, v_{i j}=w_{i}+w_{j}, 1 \leqslant i \leqslant n$, совпадают с рачиональными операторами Данкла.

\section{Список литературы}

[1] C. F. Dunkl, Trans. Amer. Math. Soc., 311:1 (1989), 167-183. [2] V. Golubeva, V. Leksin, J. Math. Sci. (New York), 98:3 (2000), 291-318. [3] Н. Бурбаки, Группъ и алгебры Ли. Элементы математики, Мир, М., 1972. [4] В. В. Мещеряков, Матем. заметки, 82:5 (2007), 709-717. [5] Дж. Харрис, Алгебрачческая геометрия, МЦНМО, М., 2006.

В. В. Мещеряков (V. V. Meshcheryakov)

Коломенский государственный педагогический институт

E-mail: metcherykov@mail.ru
Представлено Д. В. Аносовым Принято редколлегией 15.10.2008 